\title{
Associação de alterações anatomopatológicas com o Helicobacter spp. por meio de análise imuno-histoquímica em estômagos de leitões*
}

\section{Association of anatomopathological changes with the Helicobacter spp. through immunohistochemical analysis in piglets stomachs}

Renato Luiz Silveira, ${ }^{* *}$ Ana Claudia de Menezes Cruz, ${ }^{* *}$ Fabiana Batalha Knackfuss, ${ }^{* * *}$ Raphael Mansur Medina, ${ }^{* * *}$ Rachel Bittencourt Ribeiro Rodrigues, ${ }^{* * * * *}$ Mariah Bianchi Reis Gusmão Petronilha, ${ }^{* * * * *}$ Maria Aparecida da Silva, ${ }^{* * * * *}$ Talita Pinheiro Bonaparte, ${ }^{* * * * * *}$ Rita da Trindade Ribeiro Nobre Soares, ${ }^{* * * *}$ Eulógio Carlos Queiroz de Carvalho****

\section{Resumo}

O objetivo deste trabalho foi relacionar os achados anatomopatológicos das lesões gástricas subclínicas de ocorrência natural em leitões com a presença, ou não, de Helicobacter spp. por meio da imuno-histoquímica. Foram utilizados 48 leitões de linhagem genética comercial. Os animais foram adquiridos em uma granja comercial, com peso médio de 34 Kg e idade média de 79 dias; após o abate, seus estômagos foram coletados e avaliados. Avaliações histopatológicas e imuno-histoquímicas foram realizadas em amostras das regiões anatômicas aglandular e glandular. Macroscopicamente, 34 (70,83\%) leitões apresentaram lesões na região aglandular, enquanto que em 14 animais $(29,17 \%)$ não foram encontradas alterações nesta região. Dos estômagos com lesão, 14 foram classificados como grau 1, seis como grau 2 e 14 como grau 3. Microscopicamente, 44 amostras (91,66\%) apresentaram paraqueratose. Deste total, 22 apresentaram a forma discreta, 20 a moderada e dois a acentuada. Na avaliação macroscópica da porção glandular, 41 (85,4\%) animais apresentaram alteração em pelo menos uma das três regiões, e em somente sete $(14,6 \%)$ não foram encontradas lesões em nenhuma delas. Em 14 deles, houve aumento da atividade mucípara, em dois, houve erosão e, em cinco, hiperemia. As lesões na região glandular do estômago foram mais extensas no antro e no cárdia, seguidas do fundo. Em relação à análise imuno-histoquímica, 21 (43,8\%) amostras tiveram resultados negativos em todas as regiões, e 24 (50\%) foram positivas em pelo menos uma delas, sendo que nenhuma foi positiva em todas. Os achados anatomopatológicos demonstraram relação estatística com a bactéria e, sua imunomarcação não associada à lesão em certas regiões gástricas, demonstra seu caráter saprofítico e oportunista.

Palavras-chave: Helicobacter, lesões, suínos, úlcera gástrica.

\begin{abstract}
The aim of this study was to relate the anatomopathological findings of naturally occurring subclinical gastric lesions in piglets, with or without Helicobacter spp. through immunohistochemistry. Forty-eight piglets of commercial genetic lineage were used. The animals were acquired in a commercial farm, with an average weight of $34 \mathrm{~kg}$ and an average age of 79 days, and after slaughter, their stomachs were collected and evaluated. Samples from the glandular and aglandular anatomical regions were evaluated. Macroscopically, 34 (70.83\%) samples had lesions on aglandular region, while $14(29.17 \%)$ nothing had. Of the injured stomachs, 14 were classified as grade 1, six as grade 2 and 14 as grade 3. Microscopically, 44 samples (91.66\%) showed parakeratosis. Of these, 22 showed a discreet manner, 20 moderate and two severe. In the glandular region, in 41 ( $85.4 \%$ ) samples there was a change in at least one of the three regions, and only seven animals (14.6\%) showed no change in any of the three. Fourteen samples showed increased muciparous activity, two showed erosion and five hyperemia. The lesions were higher in antral regions and cardic, followed the fundus. In relation to immunohistochemistry, 21(43.8\%) samples were negative in all areas, 24 (50\%) were positive in at least one, and none were positive in all. The anatomopathological findings showed a statistical relationship with the bacteria, and its immunostaining, not associated with gastric lesions in certain regions, demonstrates its saprophytic and opportunistic character.
\end{abstract}

Keywords: Helicobacter, injuries, gastric ulcer, swine.

${ }^{*}$ Recebido em 15 de fevereiro de 2020 e aceito em 4 de junho de 2020.

"UFF - MMO/MCV/NAL - Niterói-RJ. renatosilveira@id.uff.br

"'"UNIGRANRIO - Escola de Ciências da Saúde - Faculdade de Veterinária - Duque de Caxias-RJ.

"*tUNIG - Faculdade de Veterinária - Itaperuna-RJ.

*.*'UENF - CCTA/CPGA - Campos dos Goytacazes-RJ.

*U*mES - CCAUFES/CCENS - Alegre-ES.

..m. UFBA - Faculdade de Veterinária e Zootecnia - Salvador-BA. 


\section{Introdução}

Aúlcera gástrica é uma das principais causas de morte súbita na espécie suína. Esta enfermidade apresenta caráter multifatorial, acometendo animais criados intensivamente e confinados. Devido à sua alta prevalência maiores investigações sobre os seus fatores de risco merecem ser realizadas (De Witte et al., 2019).

O aprofundamento das pesquisas sobre a participação destes fatores, além da busca de novos conhecimentos sobre a etiopatogenia do quadro, devem ser contínuos. Não obstante, o prejuízo econômico causado pelas lesões deve-se pensar, também, na questão do bemestar animal (Corrêa, 2010; Rutherford et al., 2018, Holinger et al., 2018; De Witte et al., 2019).

A bactéria Helicobacter suis tem sido associada à úlcera gástrica nos suínos e constitui grande problema para os suinocultores e a indústria suinícola em geral. Dados demonstram que as pessoas em contato estreito com os animais têm um risco maior de infecção (Baele et al., 2008), devido ao caráter zoonótico da bactéria (Vermoote et al., 2011; Loughlin et al., 2018) que já foi identificada em carcaças nos matadouros (De Cooman et al., 2014), sugerindo que o consumo de carne contaminada pode constituir uma nova via de transmissão para seres humanos (De Cooman et al., 2013).

A identificação do problema, precocemente, é de suma importância e, em decorrência disto, o objetivo deste trabalho foi relacionar os achados anatomopatológicos das lesões gástricas subclínicas, de ocorrência natural em leitões, com a presença ou não de Helicobacter spp. por meio da imuno-histoquímica.

\section{Material e métodos}

O estudo foi submetido e aprovado pela Comissão de Ética no Uso de Animais (CEUA/UFF) sob o número 372/2017. Foi realizado na Universidade Estadual do Norte Fluminense Darcy Ribeiro (UENF), em Campos dos Goytacazes-RJ. As avaliações histopatológicas foram realizadas no Laboratório de Morfologia e Patologia Animal (LMPA) da UENF. Foram utilizados 48 leitões de linhagem genética comercial com peso médio de $34 \mathrm{Kg}$, variando entre 26,4 e $40,9 \mathrm{Kg}$ e com média de 79 dias de idade, variando entre 70 e 92 dias. Foram adquiridos de uma granja comercial logo após o desmame, sendo alimentados e manejados adequadamente até a sua utilização. Depois de abatidos, tiveram seus estômagos coletados e avaliados.

$\mathrm{Na}$ avaliação macroscópica, a região aglandular foi observada quanto à sua integridade (Figura 1). Pelo fato de não produzir muco, essa região é desprotegida contra os efeitos do $\mathrm{pH}$ ácido do estômago e das enzimas no seu interior (SOBESTIANSKY e BARCELLOS, 2012) e desta forma, mais sujeita a lesões do que a região glandular.

As lesões ulcerativas foram graduadas, segundo Silveira et al. (2014; 2015) e se encontram na Tabela 1.

Amostras das regiões anatômicas aglandular (quadrilátero esofágico) e glandular (cárdica, fúndica e pilórica) foram coletadas para as avaliações histopatológicas e imunohistoquímicas (Figura 2).

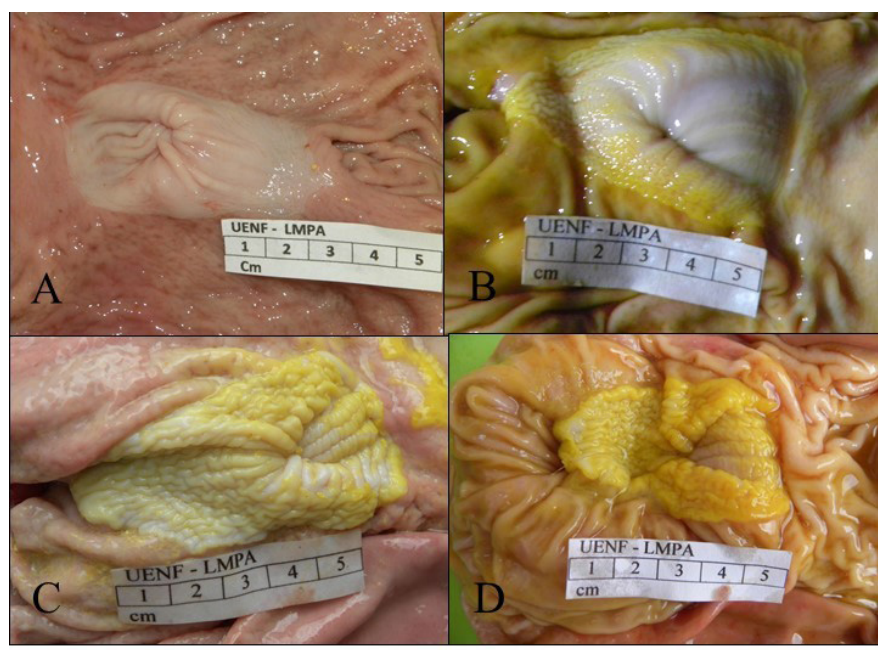

Figura 1: Fotomacrografia de estômago de leitão. Região aglandular evidenciando diferentes graus de lesão. A. Grau 0 B. Grau 1 C. 2 D. Grau 3.

Tabela 1: Classificação das alterações macroscópicas da região aglandu-lar do estômago de leitões de acordo com Silveira et al. $(2014 ; 2015)$

Escore

Região aglandular

Grau 0 Aspecto normal: liso, brilhoso e esbranquiçado.

Grau 1 Aparência parcialmente rugosa e com alguma área com pigmentação amarelada.

Grau 2 Aparência totalmente rugosa, espessada e com áreas de pigmentação amarelada

Grau 3 Aparência totalmente rugosa, espessada e com pigmentação amarelada em toda a sua superfície

Grau 4 Aparência totalmente rugosa, espessada, com pigmentação amarelada em toda a sua superfície e, ainda, mostrando algumas áreas de erosão

Grau 5 Aparência totalmente, rugosa, espessada, com pigmentação amarelada em toda a sua superfície e com áreas de ulceração

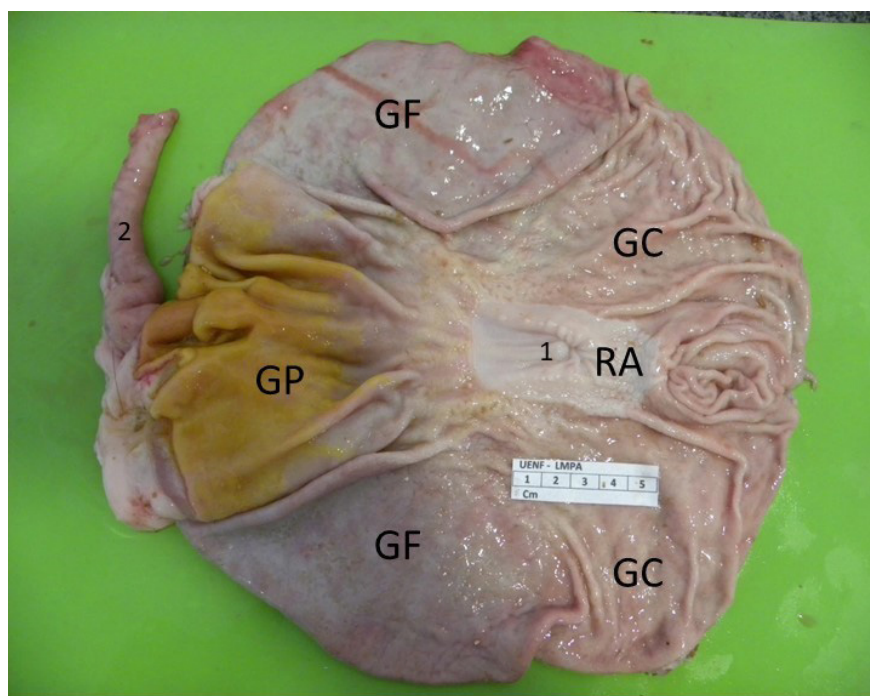

Figura 2: Fotomacrografia de estômago de leitão aberto com exposição da mucosa. Região aglandular (RA). Glândulas pilóricas (GP). Glândulas fúndicas (GF). Glândulas cárdicas (GC). Óstio cárdico (1). Duodeno (2). 
Para o diagnóstico histopatológico, as amostras coletadas foram estendidas sobre papel filtro e colocadas em frascos devidamente identificados, contendo solução de formalina neutra tamponada a $10 \%$. O processamento histotécnico foi por inclusão em parafina para coloração de rotina (HE).

Para realização da técnica de imuno-histoquímica (IHQ) foram efetuadas secções de $4 \mu \mathrm{m}$ de espessura das diferentes regiões anatômicas. Para maior aderência dos cortes histológicos, foram utilizadas lâminas silanizadas. Para desparafinização, foram realizados banhos de xilol e reidratação em banhos de álcool e água deionizada. Em seguida, realizou-se o processo de recuperação antigênica com uso do tampão citrato em banhomaria a $96^{\circ} \mathrm{C}$ por 30 minutos, seguindo para o bloqueio da peroxidase endógena à temperatura ambiente por 30 minutos, quando os espécimes foram tratados com solução alcoólica (metanol) de peróxido de hidrogênio a 10\%. Após secagem cuidadosa das lâminas, os cortes foram circundados com Dako pen $^{\circledR}$ (Dako, CA, USA) e incubados por 60 minutos com solução a $1 \%$ de soro albumina sérica bovina livre de ácidos graxos e $1 \%$ de leite em pó desnatado diluídos em Tris-NaCL para bloqueio de proteínas inespecíficas. Depois de descartada a solução de bloqueio, as secções foram incubadas overnight (17h) em câmara úmida $\left(8^{\circ} \mathrm{C}\right)$, com anticorpo policlonal anti-Helicobacter pylori (Rabbit anti-Helicobacter pylori antibody, Dako, B0471) em diluição de 1:200. No dia seguinte, as lâminas foram lavadas com solução salina e tratadas com o Kit LSAB-HRP ${ }^{+}$(Dako, CA, USA) durante 60 minutos e reveladas com Kit cromógeno DAB (Dako, CA, USA) por três minutos à temperatura ambiente. Por fim, os espécimes foram contra corados com hematoxilina de Harris, desidratados em banhos de álcoois e montados com resina sintética. Para análise das lâminas, foi usada a microscopia óptica de luz.

A IHQ é baseada na detecção de antígeno por um anticorpo, mono ou policlonal. Neste estudo, o uso do anticorpo policlonal foi em função de ser utilizado para o diagnóstico da helicobacteriose em outras espécies no hospital universitário.

Para os controles positivo e negativo, foram utilizadas amostras anteriormente testadas (SILVEIRA et al., 2014).

A fim de se analisar a existência de diferença estatística significativa entre o número de animais positivos e negativos dentro de cada porção: aglandular e glandular (Antro, Cárdica e Fundica) foi realizado o teste do Quiquadrado, considerando-se $5 \%$ de probabilidade de erro. Para esta análise foi utilizado o programa computacional SPSS 23.0.

\section{Resultados e discussão}

Segundo De Witte et al. (2018), ao contrário das outras espécies domésticas, as lesões gástricas dos suínos acontecem, na maioria das vezes, na região aglandular do estômago, o que pôde ser evidenciado neste estudo.

Macroscopicamente, 34 (70,83\%) leitões apresentaram lesões na região aglandular, enquanto que em 14 animais $(29,17 \%)$ não foram encontradas alterações nesta região (Tabela 1). Este resultado coincide com os achados de Proietti et al., 2010;
Swaby e Gregory, 2012, Marchini et al., 2017, que relataram a presença de algum tipo de lesão nesta região em mais de $70 \%$ dos estômagos avaliados. Entretanto, valores inferiores foram encontrados por outros autores (Silveira et al., 2014, Herskin et al., 2016; Omotosho et al., 2016).

Dos 34 estômagos que apresentaram lesões nesta região, 14 foram classificados como grau 1, seis como grau 2 e 14 como grau 3. Em pesquisas realizadas com animais em idade de abate, Zhu et al. (2010) em 137 animais com lesão, encontraram $32 \mathrm{com}$ o grau 1, 33 com o grau 2, $25 \mathrm{com}$ o grau 3 e $47 \mathrm{com}$ o grau 4. Swaby e Gregory (2012), em 9827 animais, encontraram $79,5 \%$ com lesões, sendo $30,3 \%$ com graus mais altos do que este estudo.

Microscopicamente, 44 amostras (91,66\%) apresentaram paraqueratose. O epitélio normal da região aglandular é liso, plano e brilhante, e facilmente diferenciado da mucosa glandular ao redor. Acredita-se que costumeiramente o progresso dessas lesões se dá a partir de paraqueratose que causa espessamento, aspecto irregular, fissuras e descamações que resultam em erosões e eventualmente ulcerações (THOMSON e FRIENDSHIP, 2019). A paraqueratose (Figura 3) é a resposta a um processo irritativo da mucosa, formada de epitélio pavimentoso estratificado corneificado. Esta irritação leva a uma hiperplasia dos queratinócitos superficiais que se apresentam com núcleos picnóticos (necrosados) (Figura 3) nas camadas córneas que se descamam (SILVEIRA et al., 2015). Estudando animais na idade de abate, Tamiasso et al. (2017) encontraram além de paraqueratose, ulcerações e infiltrado inflamatório mononuclear, alterações também observadas neste estudo.

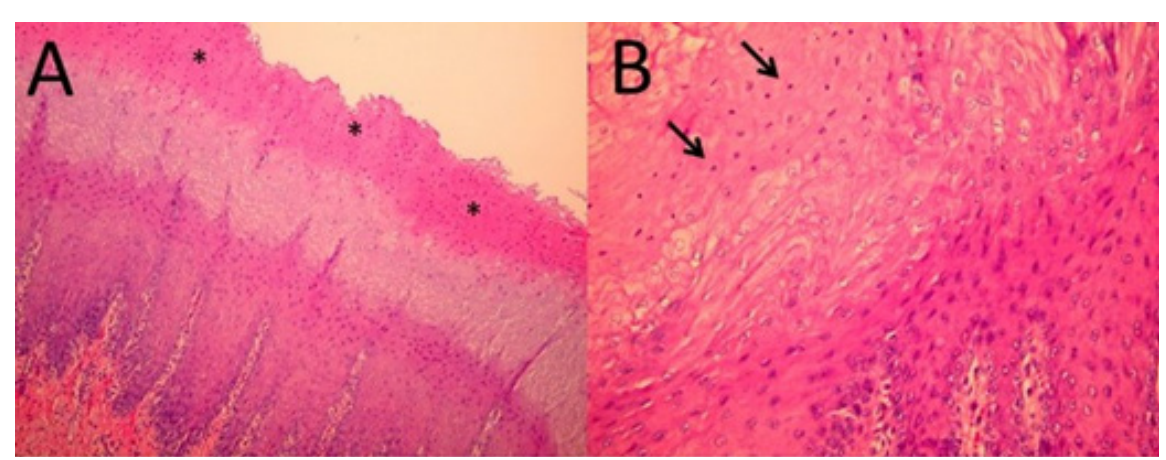

Figura 3: Fotomicrografia. Estômago de leitão. H.E. A. Região aglandular com paraqueratose Figura 3: Fotomicrografia. Estômago de leitão. H.E. A. Região aglandular com paraqueratose
(asteriscos) (Obj. 10x). B. Estrato paraqueratótico com núcleos inviáveis (picnóticos - setas) (Obj. 20x).

Os resultados encontrados estão um pouco acima dos $77,6 \%$ descritos por Yamasaki et al. (2009) e dos 75\% observados por Marruchella et al. (2004), e bem acima dos 55\% de Silveira et al. (2014). Das 44 amostras com lesão, 22 apresentaram uma forma discreta de paraqueratose, 20 moderada e duas acentuada.

$\mathrm{Na}$ avaliação macroscópica da porção glandular do estômago dos leitões, 41 (85,4\%) animais apresentaram alteração em pelo menos uma das três regiões, e em somente sete $(14,6 \%)$ não foram encontradas lesões em nenhuma delas. Em 50 leitões, houve formação de catarro. Em 14 deles, houve aumento da atividade mucípara, em dois, houve erosão e, em cinco, hiperemia. Bracarense et al. (2013) indicam, em seu estudo, que a infecção por Helicobacter spp. aumenta significativamente as lesões nesta região. 
As lesões na região glandular do estômago foram mais extensas no antro e no cárdia, seguidas do fundo. Contrariando, Silva et al. (2001) e Silveira et al. (2014) observaram um maior acometimento da região cárdica, seguida da antral e fúndica. Nas regiões das glândulas pilóricas e cárdicas, 29 animais apresentaram alteração e 19 não. Na região das glândulas fúndicas, 13 apresentaram alteração e 35 não.

Em relação à análise imuno-histoquímica, 21 (43,8\%) estômagos tiveram resultados negativos em todas as regiões, e $24(50 \%)$ positivos em pelo menos uma delas, sendo que nenhum foi positivo em todas. Szeredi et al. (2005) em seus estudos encontraram $85,4 \%$ de positividade em todas as regiões, já Yamasaki et al. (2006) relataram $52,5 \%$ dos animais estudados como negativos e $47,5 \%$ como positivos.

Neste estudo foi utilizado o anticorpo policlonal anti-Helicobacter pylori visando a identificação de Helicobacter spp., mas estudos sugerem que o agente envolvido na etiologia das úlceras em suínos seja a bactéria Helicobacter suis. Apesar disto, faltam dados certeiros sobre os agentes envolvidos. Este fato, associado ao possível impacto da infecção bacteriana na sanidade dos rebanhos, ao potencial para causar doença no homem e à possibilidade de redução nos lucros, justifica o interesse para continuar investigando o tema (TAMIASSO et al., 2017).

$\mathrm{Na}$ região aglandular, dois animais apresentaram resultado positivo e 38 negativo. Segundo Tamiasso et al. (2017), raramente se encontra a bactéria nesta região, desta forma, as lesões nesta porção do estômago aparentemente não possuem relação com a presença de Helicobacter spp. Na região do antro, foram encontrados dez positivos e 38 negativos. Na região das glândulas cárdicas, 18 foram positivos e 30 negativos (Figura 4). Na região das glândulas fúndicas, dois foram positivos e 46 negativos. Silveira et al. (2014) encontraram 10 animais positivos e 10 negativos na região do antro. Na região das glândulas cárdicas foram encontrados três positivos e 17 negativos. Na região das glândulas fúndicas dois animais foram positivos e 18 negativos.

Foram observadas diferenças estatísticas significativas quando as frequências de amostras positivas, com relação a presença da bactéria, foram comparadas com as negativas na região antral $\left(X^{2}=9.01\right.$, $p=0,0109)$. Resultado semelhante foi observado na região das glândulas fúndicas $\left(x^{2}=14.609, p=0,0056\right)$ (Tabela 1). Entretanto, não foram observadas diferenças estatísticas significativas entre cárdicas (Obj. 40x). o número de positivos e negativos nas regiões aglandular $\left(x^{2}=1.49, p=0,6844\right)$ e glandular cárdica $\left(x^{2}=1.617, p=0,6556\right)$. Resultado semelhante foi encontrado por De Witte et al. (2017), que observaram uma prevalência maior da bactéria na região pilórica.

Também por meio da IHQ, Szeredi et al. (2005) encontraram maior infecção na região cárdica, seguida da pilórica e fúndica. Uma das vantagens desta técnica é o diagnóstico de cepas com formas, predominantemente, cocóides que, nas colorações de rotina, são mais difíceis de serem observadas (McNulty et al., 2011).

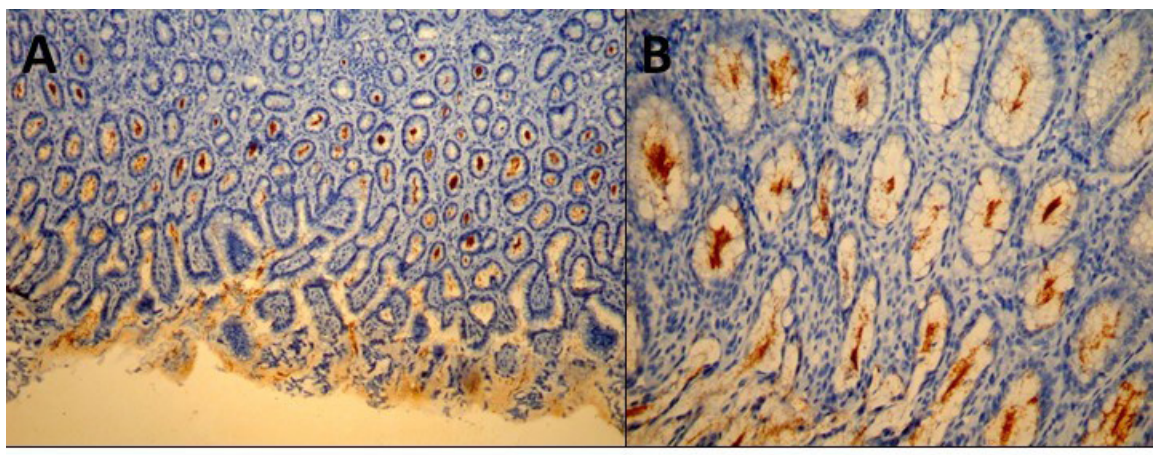

Figura 4: Fotomicrografia de estômago de leitão revelando imunomarcação com anticorpo antiHelicobacter pylori. HE/IHQ. A. Região de glândulas pilóricas (Obj. 10x) B. Região de glândulas

Tabela 1: Presença de Helicobacter spp. na mucosa gástrica de leitões com diferentes graus de lesão, detectada por meio da imuno-histoquímica

\begin{tabular}{|c|c|c|c|c|c|c|c|}
\hline & $\begin{array}{c}\text { Presença de } \\
\text { Helicobacter } \\
\text { spp. }\end{array}$ & Grau 0 & Grau 1 & Grau 2 & Grau 3 & & Total \\
\hline Parte & Positivo & 01 & 00 & 00 & 01 & & 02 \\
\hline Aglandular & Negativo & 13 & 14 & 06 & 13 & & 46 \\
\hline \multirow[t]{2}{*}{ NS } & Total & 14 & 14 & 06 & 14 & & 48 \\
\hline & & Normal & $\begin{array}{l}\text { Atividade } \\
\text { Mucípara }\end{array}$ & Erosão & Hiperemia & Catarro & \\
\hline Parte & Positivo & 00 & 01 & 00 & 00 & 09 & 10 \\
\hline $\begin{array}{c}\text { Glandular } \\
\text { (Antro) }\end{array}$ & Negativo & 19 & 04 & 00 & 00 & 15 & 38 \\
\hline \multirow[t]{2}{*}{$p<0.05$} & Total & 19 & 05 & 00 & 00 & 24 & 48 \\
\hline & & Normal & $\begin{array}{l}\text { Atividade } \\
\text { Mucípara }\end{array}$ & Erosão & Hiperemia & Catarro & \\
\hline Parte & Positivo & 07 & 02 & 00 & 00 & 09 & 18 \\
\hline $\begin{array}{l}\text { Glandular } \\
\text { (Cárdica) }\end{array}$ & Negativo & 12 & 06 & 01 & 00 & 11 & 30 \\
\hline \multirow[t]{2}{*}{ NS } & Total & 19 & 08 & 01 & 00 & 20 & 48 \\
\hline & & Normal & $\begin{array}{l}\text { Atividade } \\
\text { Mucípara }\end{array}$ & Erosão & Hiperemia & Catarro & \\
\hline \multirow{2}{*}{$\begin{array}{c}\text { Parte } \\
\text { Glandular } \\
\text { (Fúndica) }\end{array}$} & Positivo & 00 & 00 & 00 & 00 & 02 & 02 \\
\hline & Negativo & 35 & 01 & 01 & 05 & 04 & 46 \\
\hline$p<0.05$ & Total & 35 & 01 & 01 & 05 & 06 & 48 \\
\hline
\end{tabular}

NS = não significativo; $p<0.05=$ existem diferenças estatísticas significativas pelo teste de Quiquadrado entre a frequência observada de animais positivos e negativos, dentro da porção avaliada no teste de imuno-histoquímica. 


\section{Conclusões}

Os achados anatomopatológicos demonstraram relação estatística com a bactéria e, sua imunomarcação não associada

\section{Referências}

BAELE, M.; DECOSTERE, A.; VANDAMME, L.; CEELEN, L.; HELLEMANS, A.; MAST, J.; CHIERS, K.; DUCATELLE, R.; HAESEBROUCK, F. Isolation and characterization of Helicobacter suis sp. nov. from pig stomachs. International Journal of Systematic and Evolutionary Microbiology, v.58, n.6, p.1350-1358, 2008.

BRACARENSE, A.P.F.R.L.; YAMASAKI, L.; SILVA, O.; OLIVEIRA, R.L.; ALFIERI, A.A. Helicobacter spp. Infection induces changes in epitelial proliferation and E-cadherin expression in the gastric mucosa of pigs. Journal of Comparative Pathology, v.149, n.4, p.402-409, 2013.

CORRÊA, A.M.R.C. Úlceras gástricas em suínos. Informativo Técnico DPA-RS, v.1, n.6, Setembro, 2010.

DE COOMAN, L.; FLAHOU, B.; HOUF, K.; SMET, A.;

DUCATELLE, R.; PASMANS, F.; HAESEBROUCK, F. Survival of Helicobacter suis bactéria in retail pig meat. International Journal of Food Microbiology, v.166, n.1, p.164-167, 2013.

DE COOMAN, L.; HOUF, K.; SMET, A.; FLAHOU, B.; DUCATELLE, R.; PASMANS, F.; HAESEBROUCK, F. Presence of Helicobacter suis on pork carcasses. International Journal of Food Microbiology, v.187, n.18, p.73-76, 2014.

DE WITTE, C.; DEMEYERE, K.; DE BRUYCKERE, S.; TAMINIAU, B.; DAUBE, G.; DUCATELLE, R.; MEYER, E.; HAESEBROUCK, F.

Characterization of the non-glandular gastric region microbiota in Helicobacter suis-infected versus non-infected pigs identifies a potential role for Fusobacterium gastrosuis in gastric ulceration. Veterinary Research, v.50, n.39, p.1-18, 2019.

DE WITTE, C.; DEVRIENDT, B.; FLAHOU, B.; BOSSCHEM, I.; DUCATELLE, R.; SMET, A.; HAESEBROUCK, F. Helicobacter suis induces changes in gastric inflammation and acid secretion markers in pigs of different ages. Veterinary Research, v.48, n.34, p.1-13, 2017.

DE WITTE, C.; DUCATELLE, R.; HAESEBROUCK, F. The role of infectious agents in the development of porcine gastric ulceration. The Veterinary Journal, v.236, June, p.56-61, 2018.

THOMSON, J.R.; FRIENDSHIP, R.M. Digestive system. In: Diseases of swine. Hoboken. Wiley Blackwell. Cap. 15. 1108p. 2019, p.234-263.

HERSKIN, M.S.; JENSEN, H.E.; JESPERSEN, A.; FORKMAN, B.; JENSEN, M.B.; CANIBE, N.; PEDERSEN, L.J. Impact of the amount of straw provided to pigs kept in intensive production conditions on the occurrence and severity of gastric ulceration at slaughter. Research in Veterinary Science. v.104, February, p.200-206, 2016.

HOLINGER, M.; BARBARA FRÜH, B.; STOLL, P.; KREUZER, M.; HILLMANN, E. Grass silage for growing-finishing pigs in addition to straw bedding: Effects on behaviour and gastric health. Livestock Science, v.208 (December), p.50-58, 2018. à lesão em certas regiões gástricas, demonstra seu caráter saprofítico e oportunista.

LOUGHLIN, V.H.M.; NAVARRO, O.E.; MARTÍNEZ, R.A.; GROSSO, M.C.; SAVINO, F.; RITTA, L.; LEANDRO, M.P.; VEEN, V.D.; GIMÉNEZ, S.R.; SORIA, M.E.M.; CRISTOFOLINI, A.; MERKIS, C.I. Determinação de Helicobacter Spp. no estômago de suínos da zona de Río Cuarto, Córdoba, Argentina. Academia Journal of Biotechnology, vol.7, n.1, p.8-13, 2018.

MARCHINI, C.F.P.; MARTINS, P.M.; RABELO, R.N. Prevalence of gastric lesion in pigs. Investigação, v.16, n.8, p.50-55, 2017.

MARRUCHELLA, G.; DI LEONARDO, M.; DI GUARDO, G.; ROMANUCCI, M.; MRA, M.; TISCAR, P.G.; MOSCA, F.; DELLA SALDA, L. Heat shock proteins (HSPs) 27,72 and 73 in normal and pré-ulcerative mucosa of the gastric pars oesophagea in swine. Journal of Comparative Pathology, v.131, n.1, p.10-17, 2004.

McNULTY, C.A.M.; LEHOURS, P.; MÉGRAUD, F. Diagnosis of Helicobacter pylori infection. Helicobacter, v.16, sup.1, p.10-18, 2011.

OMOTOSHO, O. O.; EMIKPE, B.O.; LASISI, O.T.; JARIKRE, T.A. Prevalence, distribution and pattern of gastric lesions in slaughtered pigs in south-western Nigeria. Onderstepoort Journal of Veterinary Research, v.83, n.1, p.1-6, 2016.

PROIETTI, P.C. ; BIETTA, A. ; LEPRI, E. ; DAVIDSON, I.; FRANCIOSINI, M.P. Detection of Helicobacter spp. in gastric, fecal and saliva samples from swine affected by gastric ulceration. Journal of Veterinary Sciense, v.11, n.3, p.221-225, 2010.

RUTHERFORD, K.M.D.; THOMPSON, C.S.; THOMSON, J.R.; LAWRENCE, A.B.; NIELSEN, E.O.; BUSCH, M.E.; HAUGEGAARD, S.; SANDØE, P. A study of associations between gastric ulcers and the behaviour of finisher pigs. Livestock Science, v.212 (June), p.45-51, 2018.

SILVA, J.C.P.; SANTOS, J.L.; BARBOSA, A.J.A. Gastrite em suínos: frequência, relação com a úlcera gástrica e com a densidade de células endócrinas do estômago. Revista Brasileira de Ciência Veterinária, v.8, n.1, p.40-43, 2001.

SILVEIRA, R.L.; CRUZ, A.C.M.; MEDINA, R.M.; CÂMARA FILHO, J.A.; ABÍLIO, E.J.; CARVALHO, E.C.Q. Lesões pré-ulcerativas gastroesofágicas em miniporcos. Revista Brasileira de Ciência Veterinária. v.22, n.3-4, p.160-164, 2015.

SILVEIRA, R.L.; CRUZ, A.C.M.; WEBER, F.A.G.C.; DEGANI, V.A.N.; ABÍLIO, E.J.; CARVALHO, E.C.Q. Helicobacteriose em leitões: imuno-histoquímica em amostras colhidas por meio de gastroscopia. Arquivo Brasileiro de Medicina Veterinária e Zootecnia, v.66, n.6, p.1681-1686, 2014.

SOBESTIASKY, J.; BARCELLOS, D. Doenças dos Suínos. 2ed., Goiânia-GO. Cânone Editorial, 2012, 960p.

SWABY, H.; GREGORY, N.G. A note on frequency of gastric ulcers detected during post mortem examination at a pig abattoir. Meat Sciense, v.90, n.1, p.269-271, 2012. 
SZEREDI, L. ; PALKOVICS, G. ; SOLYMOSI, N. ; TEKES, L.; MEHESFALVI, J. Study on the role of gastric Helicobacter infection on gross pathological and hitological lesions of the stomach in finishing pigs. Acta Veterinaria Hungarica, v.53, n.3, p.371-383, 2005.

TAMIASSU, N.V.; COSME, J.C.; FERREIRA, M.F.; NUNES, L.C. Ausência de Helicobacter spp. em lesões gástricas de suínos pelo método Warthin-Starry. Veterinária e Zootecnia, v.24, n.2, p.336-344, 2017.

VERMOOTE, M.; VANDEKERCKHOVE, T.T.M.; FLAHOU, B.; PASMANS, F.; SMET, A.; DE GROOTE, D.; VAN CRIEKINGE, W.; DUCATELLE, R.; HAESEBROUCK, F. Genome sequence of Helicobacter suis supports its role in gastric pathology. Veterinary Research, v.42, n.51, p.1-9, 2011.
YAMASAKI, L.; ASSIS, F.M.S.; ROSSETO, V.J.V.; BRACARENSE, A.P.F.R.L. Lesões gástricas em suínos: Ocorrência e relação com o gênero, peso ao abate e presença de Helicobacter spp. Semina Ciências Agrárias. v.27, n.3, p.463-470, 2006.

YAMASAKI, L. ; BOSELLI-GROTTI, C.C. ; ALFIERI, A.A. ; SILVA, E.O.; OLIVEIRA, R.L.; CAMARGO, P.L.; BRACARENSE, A.P.F.R.L. Alterações histológicas da pars esophagea de suínos e sua relação com Helicobacter spp. Arquivo Brasileiro de Medicina Veterinária e Zootecnia, v.61, n.3, p.553-560, 2009.

ZHU, C.; WEY, D.; FRIENDSHIP, R. Impact of fineness of grinding of corn and high-moisture corn on growth performance, carcass characteristics and stomach lesions in liquid-fed growing-finishing pigs. In: International Pig Veterinary Society Congress. 21, 2010. Proceedings... Vancouver, Canadá. p.1062, 2010. 